\title{
Baric Shift of Phase Transition in Ammonium Sulfate Crystals
}

\author{
V.M. GABA \\ Lviv Polytechnic National University, 12 Bandera St., 79013 Lviv, Ukraine
}

\begin{abstract}
The influence of uniaxial mechanical pressures $\left(\sigma_{\mathrm{m}} \leq 200 \mathrm{bar}\right)$ on the spectral $(300-800 \mathrm{~nm})$ and temperature $(77-300 \mathrm{~K})$ dependences of the refractive indices $n_{i}$, the birefringence $\Delta n_{i}$ and the phase transition point in ammonium sulfate crystals are studied. It is established that the uniaxial pressure does not change character of the dispersion $\mathrm{d} n_{i} / \mathrm{d} \lambda$, but only its value $\lambda$ The shift of the phase transition in ammonium sulfate under the uniaxial mechanical pressure is analyzed.
\end{abstract}

PACS numbers: 77.84.Fa, 78.20.Ci, 77.80.Bh

\section{Introduction}

Ammonium sulfate (AS) $\left(\left(\mathrm{NH}_{4}\right)_{2} \mathrm{SO}_{4}\right)$ crystals are not polar at the room temperature and belong to the space group Pnma of the orthorhombic system. Below $T_{\mathrm{c}}=$ $223 \mathrm{~K}$, they undergo a ferroelectric phase transition (PT) without a change in the number of formula units per unit cell into the space group $P n 2 a_{1}[1,2]$. This PT is accompanied by a significant step in the strain, while the spontaneous polarization $P_{\mathrm{c}}$ below $T_{\mathrm{c}}$ decreases in magnitude and changes its sign near $85 \mathrm{~K}[3]$. This testifies to the presence of several temperature-dependent contributions to $P_{\mathrm{c}}$ from different sublattices [4] and a compensation of these contributions near $85 \mathrm{~K}$.

As compared with some other ferroelectrics (e.g., triglycine sulfate), the AS crystals manifest many specific properties. For example, their Curie-Weiss constant is very small $(C \approx 15.6)$ and satisfies the Curie-Weiss law only in a narrow temperature interval near $T_{\mathrm{c}}$. The $P_{\mathrm{c}}$ value at $T<T_{\mathrm{c}}\left(P_{\mathrm{c}} \approx 0.6 \mathrm{mC} / \mathrm{cm}^{2}\right)$ is also rather small and changes its sign below $85 \mathrm{~K}[5,6]$.

The unit cell of AS crystals contains four formula units, which possess two nonequivalent ammonium ions $\mathrm{NH}_{4}^{+}(1)$ and $\mathrm{NH}_{4}^{+}(2)$ in both phases. The structural studies of the para- and ferroelectric phases of AS crystals have revealed ordered elements such as $\mathrm{NH}_{4}^{+}(1), \mathrm{NH}_{4}^{+}(2)$ and $\mathrm{SO}_{4}$ groups. It has been concluded that $\mathrm{SO}_{4}$ tetrahedra in the paraelectric phase do not have two equilibrium positions and move in a single-minimum potential $[7,8]$.

Studies of the influence of uniaxial pressures on the spectral and temperature dependences of optical birefringence $\Delta n_{i}$ and the refractive indices $n_{i}$ of AS and of materials isomorphic to AS have shown significant baric sensitivity of electron subsystem of these crystals, which has been revealed in considerable shift of the positions of effective bands of ultraviolet and infrared oscillators in their energy spectrum [9-12].

The aim of this work is to study the effect of uniaxial mechanical pressure on the temperature dependences of the refractive indices $n_{i}$ and the position of the PT point.

\section{Experimental procedure}

AS crystals were grown from aqueous stoichiometric solution using a slow cooling technique. They had the form of rhombic prisms with a large number of faces. Typical dimensions of the plates were $100 \mathrm{~mm}^{2}$ in area and $1 \mathrm{~mm}$ in thickness. No twinning was visible under optical microscopic observation. The samples were placed into a cryostat filled with helium gas, enabling the temperature to be kept uniform to within $0.01 \mathrm{~K}$.

The dispersion $n_{i}(\lambda)$ was studied in the spectral range 300-800 nm after measuring changes in the interference pattern of polarized rays, which was detected with the aid of a spectral device DFS-8 equipped with a modernized input unit. Upon passing of white light through such a system, the interference pattern arises in the focal plane of a spectrometer. The spectral minima of the pattern satisfy the condition

$$
n_{i}=1+\frac{k \lambda}{d}
$$

where $k$ is the order of the interference maximum, $d-$ the sample thickness along the path, and $n_{i}$ - the refractive index for the principal direction $i$.

Direct study of the changes in the refractive indices of samples under pressure is a difficult technical problem. In this work, the baric changes in the principal refractive indices $n_{i}$ of our crystals were analyzed while determining piezooptic constants $\pi_{i m}$ and calculating subsequently the changes $n_{i}(\sigma)$ using the formula

$$
\begin{aligned}
& n_{i}(\lambda, \sigma, T) \\
& \quad=n_{i 0}(\lambda, T)-\frac{1}{2} \pi_{i m}(\lambda, T) \times \sigma_{\mathrm{m}} n_{i 0}^{3}(\lambda, T),
\end{aligned}
$$

where $n_{i 0}$ denotes the refractive index of mechanically free crystal.

This method makes it possible to investigate behavior of the refractive indices under different pressures (especially the changes in $n_{i}$ under $\sigma_{i}$ ) for wide spectral and temperature ranges. The well-known direct interference optical method [9] makes it possible to investigate such 
dependences for one wavelength only and, moreover, it is very difficult to implement in practice.

\section{Experimental results and discussion}

It has been established (see Fig. 1) that, in the temperature range of the paraphase $(T>223 \mathrm{~K})$, the principal refractive indices increase almost linearly with decreasing temperature: $\mathrm{d} n_{x} / \mathrm{d} T=-5.1 \times 10^{-5} \mathrm{~K}^{-1}, \mathrm{~d} n_{y} / \mathrm{d} T=$ $-2.9 \times 10^{-5} \mathrm{~K}^{-1}$, and $\mathrm{d} n_{z} / \mathrm{d} T=-3.8 \times 10^{-5} \mathrm{~K}^{-1}$. At $T=223 \mathrm{~K}$, the phase transition (PT) occurs, which is accompanied by a stepwise decrease in the refractive indices $\left(\delta n_{z} \approx 6.3 \times 10^{-4}, \delta n_{x} \approx 5.9 \times 10^{-4}\right.$ and $\left.\delta n_{y} \approx 4.1 \times 10^{-4}\right)$. Cooling in the polar phase leads to some decrease in $n_{i}$; In the temperature range of $77-160 \mathrm{~K}$ the values $n_{i}$ tend to constant values and then hardly change with temperature $\left(\mathrm{d} n_{i} / \mathrm{d} T \approx 0\right)$. The action of uniaxial pressures along different principal directions leads to an increase in $n_{i}$. However, the character of temperature changes $\mathrm{d} n_{i} / \mathrm{d} T$ and the values of steps at the PTs $\left(\delta n_{z} \approx 6.0 \times 10^{-4}, \delta n_{x} \approx 5.1 \times 10^{-4}\right.$ and $\delta n_{y} \approx 5.2 \times 10^{-4}$ ) do not change radically. A significant change is observed in the position of the PT point: the uniaxial pressure $\sigma_{x}$ shifts the PT point towards higher temperatures $\left(T_{\mathrm{c}}^{x}=228.0 \mathrm{~K}\right)$, while the pressures along the $z$ and $y$ axes shift it towards lower temperatures $\left(T_{\mathrm{c}}^{z}=216.5 \mathrm{~K}\right.$ and $\left.T_{\mathrm{c}}^{y}=218.8 \mathrm{~K}\right)$.

The baric coefficients of the shift of PT point have the following values: $\partial T_{\mathrm{c}} / \partial \sigma_{x}=+0.024 \mathrm{~K} / \mathrm{bar}, \partial T_{\mathrm{c}} / \partial \sigma_{y}=$ $-0.021 \mathrm{~K} / \mathrm{bar}$, and $\frac{\partial T_{\mathrm{c}}}{\partial \sigma_{z}}=-0.046 \mathrm{~K} / \mathrm{bar}$. The total coefficient of baric shift of the PT point (if we suggest that it is the analogue of hydrostatic pressure) is equal to $\partial T_{\mathrm{c}} / \partial p=-0.043 \mathrm{~K} / \mathrm{bar}$. These data are in $\operatorname{good}$ agreement with the results of differential thermal analysis of the $p-T$ phase diagram of AS crystals under hydrostatic pressures up to $p \approx 6 \mathrm{kbar}$, which have established that the hydrostatic pressure leads to a linear shift of $T_{\mathrm{c}}$ $(-4.1 \mathrm{~K} / \mathrm{kbar})$.

We relate the baric shift of the PT point of AS crystals under the action of pressures to the crystal structure changes. It is known that corresponding structure at the room temperature is described by the space symmetry Pnma. In Fig. 2 we present projection of the AS structure onto the $y z$ plane. Tetrahedral groups $\mathrm{SO}_{4}$ are somewhat distorted, and two tetrahedral $\mathrm{NH}_{4}$ behave as two different dipoles, $\mathrm{NH}_{4}(\mathrm{I})$ and $\mathrm{NH}_{4}(\mathrm{II})$.

The projection of hydrogen atom $\mathrm{H}(\mathrm{I})$ is normal to the $x y$ plane, in which the atoms $\mathrm{H}(\mathrm{II}), \mathrm{H}(\mathrm{III})$ and $\mathrm{H}(\mathrm{IV})$ are positioned. The hydrogen atom $\mathrm{H}(\mathrm{I})$ is the most unstable in the tetrahedral $\mathrm{NH}_{4}$. Therefore, the motion of the bond $\mathrm{N}-\mathrm{H}(\mathrm{I})$ can be qualitatively related to the deformed tetrahedron $\mathrm{NH}_{4}$. The three hydrogen atoms are normal to the $x y$ plane and oscillate near the position of equilibrium.

Generally, the AS crystal structure in the paraelectric phase could be considered as a partially disordered state with respect to small rotations of the groups $\mathrm{SO}_{4}, \mathrm{NH}_{4}(\mathrm{I})$ and $\mathrm{NH}_{4}(\mathrm{II})$, mainly around the pseudo-hexagonal $z$ axis.

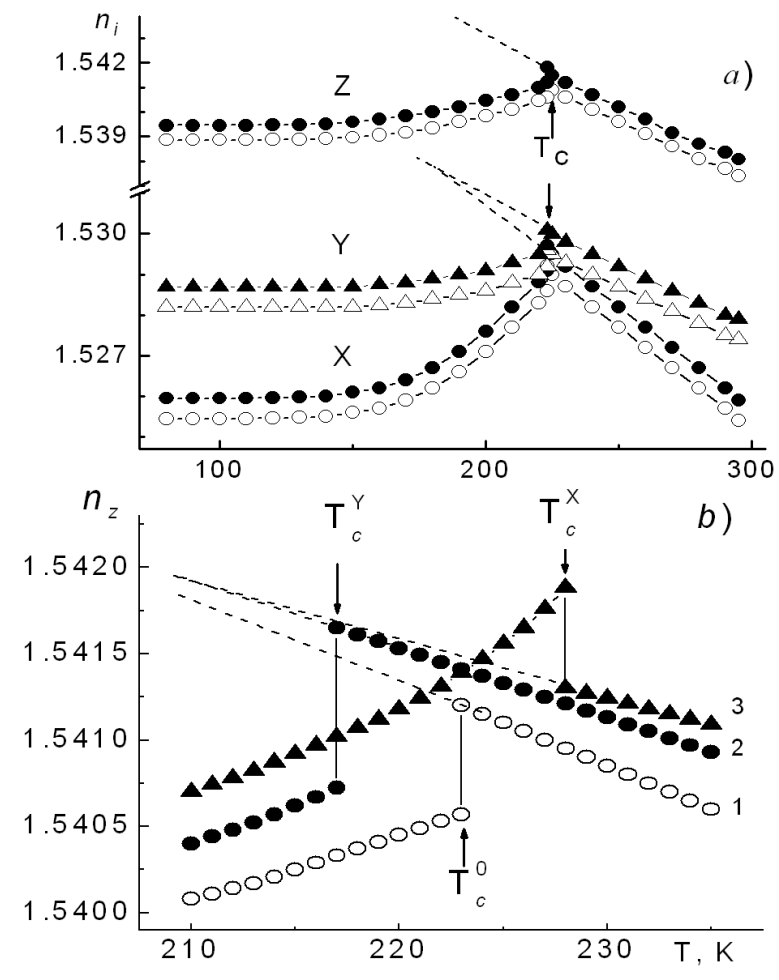

Fig. 1. Temperature changes of the refractive indices $n_{i}$ (a) and $n_{z}$ (b) of mechanically free (open circles) and uniaxially stressed (full circles and triangles) of the AS crystals in the PT region: 1 - mechanically free crystal, $\sigma=0 ; 2-\sigma_{y}=150$ bar; $3-\sigma=150$ bar.

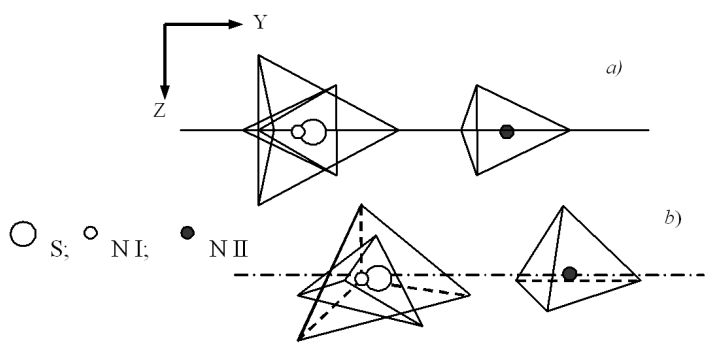

Fig. 2. Projection of elementary cell of the AS crystals onto the $y z$ plane in the paraelectric (a) and ferroelectric (b) phases.

The ordering of structural elements in the course of ferroelectric transition consists, in particular for sulphate groups, in small displacements accompanied by rotations of the groups [7]. The temperature dependence of the rotation angles for the $\mathrm{SO}_{4}$ groups and the ordering parameter for the ammonium groups in one of the orientations correlate with each other. At the same time, the authors [7] have suggested that the $\mathrm{SO}_{4}$ ions are not elements of the structure which are being ordered, because the rotation angles of the group depend on the temperature.

It is reckoned that the PT in the AS crystal is a transition of particular type [13]. The PT in the AS is mainly 
defined by the $\mathrm{SO}_{4}$ groups which change collectively their orientation, positions and the distortions at $T_{\mathrm{c}}$, thus determining a partial ordering of the ammonium groups which conserve a possibility to reorient below $T_{\mathrm{c}}$. In this case, the number of rotations of tetrahedra $\mathrm{SO}_{4}^{2-}$ around all three axes of the structure grows, but this occurs mainly around the $x$ axis. Then the tetrahedron is "frozen" in one of the orientational states.

If the influence of external field coincides with that caused by decreasing temperature, then the thermodynamic equilibrium occurs at higher temperatures. In the opposite case, the PT temperature would shift towards lower temperatures. Since the PT is accompanied by increasing rotation of the tetrahedron $\mathrm{SO}_{4}^{2-}$ around the $z$ axis in the ferroelectric phase, the uniaxial pressure along this axis "clamps" the tetrahedron, i.e. it decelerates its rotation to the symmetric state and, therefore, causes a decrease in the temperature required for the destruction of this state. Therefore lower temperatures are needed for the PT to occur under this uniaxial pressure.

A rotation of $\mathrm{SO}_{4}^{2-}$ tetrahedron in the AS crystal happens in the $x y$ plane under the PT. A shift of the PT towards lower temperatures under the action of the pressure $\sigma_{y}$ is obviously associated with the fact that the direction of $\sigma_{y}$ is opposite to that of the rotation of the tetrahedron $\mathrm{SO}_{4}^{2-}$ around the $z$ axis. In other words, the pressure $\sigma_{y}$ "decelerates" rotation of the tetrahedron and, correspondingly, the condition of thermodynamical equilibrium is satisfied at lower temperatures. The action of the pressure $\sigma_{x}$ coincides with that of the rotation of $\mathrm{SO}_{4}^{2-}$ tetrahedron at the PT. Therefore, it "accelerates" the transition into the ferroelectric phase; In this respect, the PT would occur at higher temperatures which "compensate" the mechanical ordering.

\section{References}

[1] J.A. Mc. Ginnety, Acta Crystallogr. b 28, 2845 (1972).

[2] A.J. van den Berg, F. Tuinstra, Acta Crystallogr. B 34, 3177 (1978).

[3] M. Miyake, S. Iwai, Phys. Chem. Miner. 7, 211 (1981).

[4] H. Arnold, W. Kurtz, Ferroelectrics 25, 557 (1980).

[5] A. Onodera, H. Fujishita, Y. Shiozaki, Solid State Commun. 27, 463 (1978).

[6] A. Bard, S. Awad, J. Phys. Chem. Solids 45, 351 (1984).

[7] A. Sawada, Y. Takagi, Y. Ishibashi, J. Phys. Soc. Japan 34, 748 (1973).

[8] A. Onodera, Y. Sugata, Y. Shiozaki, Solid State Commun. 27, 243 (1988).

[9] V.Yo. Stadnyk, M.O. Romanyuk, Ferroelectrics 317, 255 (2005).

[10] V.Yo. Stadnyk, M.O. Romanyuk, B.V. Andrievskii, M.R. Tuzyak, Crystallogr. Rep. 54, 313 (2009).

[11] V.Yo. Stadnyk, O.S. Kushnir, R.S. Brezvin, V.M. Gaba, Opt. Spectrosc. 106, 614 (2009).

[12] V.Yo. Stadnyk, M.O. Romanyuk, M.R. Tuzyak, V.Y. Kurlyak, Ukr. J. Phys. 54, 587 (2009).

[13] Y.S. Jain, P.K. Baipai, R. Bhattacharjee, J. Phys. C, Solid State Phys. 19, 3789 (1986). 